\title{
SUPPORTING INFORMATION \\ Principles of Dynamic Heterogeneous Catalysis: Surface Resonance and Turnover Frequency Response
}

\author{
M. Alexander Ardagh ${ }^{1,2}$, Omar A. Abdelrahman ${ }^{2,3}$, Paul J. Dauenhauer ${ }^{1,2^{*}}$
}

${ }^{1}$ University of Minnesota, Department of Chemical Engineering and Materials Science, 421 Washington Ave SE, Minneapolis, MN 55455

${ }^{2}$ Catalysis Center for Energy Innovation, University of Delaware, 221 Academy Street, Newark, DE 19716

${ }^{3}$ University of Massachusetts Amherst, Department of Chemical Engineering, 159 Goessmann Laboratory, 686 North Pleasant Street, Amherst, MA 01003.

*Corresponding author: hauer@umn.edu

$$
\begin{gathered}
\text { \# of Figures: } 9 \\
\text { \# of Tables: } 1 \\
\text { \# of Equations: } 0
\end{gathered}
$$

Table of Contents:

Section S1. Matlab 2017b and 2018b Code

- CSTR Model

- Volcano Plot Generator

- Dynamic Catalysis Shell Code for Square, Triangle, and Sawtooth Waveforms

- Dynamic Catalysis Shell Code for Sinusoidal Waveform

\section{Section S2. Matlab ODE Solver Performance}

- ODE45

- ODE15s

- ODE23s

- ODE23t

- ODE23tb

Section S3. Static Catalysis Time on Stream Data

- Example at $1 \%$ Yield of B

\section{Section S4. Dynamic Catalysis Time on Stream Data}

- Square Waveform:
○ $0.001 \mathrm{~Hz}$
○ $0.25 \mathrm{~Hz}$
○ $10 \mathrm{~Hz}$
- $1000 \mathrm{~Hz}$

- $10 \mathrm{~Hz}$ Oscillation Frequency:

- Square Waveform

- Sinusoidal Waveform

- Triangle Waveform

- Sawtooth Waveform

Section S5. Figure 3 Heatmap Data 


\section{Section S1. Matlab 2017b and 2018b Code}

\section{$\underline{\text { CSTR Model }}$}

$\%$ CSTR model

$\%$ Description:

$\%$ Continuously Stirred Tank Reactor with overall reaction A -> B.

$\%$ The number of catalytic active sites is the control.

function $\mathrm{xdot}=\operatorname{cstr} 1(\mathrm{t}, \mathrm{x})$

global T Rg Caf Cbf V u deltE0 q

$\%$ Volcano parameters

$\%$ Binding energies

BEAeV0 $=1.3 ; \%$ eV

$\mathrm{BEA} 0=\mathrm{BEAeV0} * 96.485 \mathrm{e} 3 ; \% \mathrm{~J} / \mathrm{gmol}$

$\mathrm{BEBeV0}=1.0 ; \% \mathrm{eV}$

BEB $0=$ BEBeV0*96.485e3; \% J/gmol

$\%$ Correlation between $\mathrm{BE} \mathrm{A}$ and $\mathrm{BE} \mathrm{B}$

$\mathrm{dCorr} \mathrm{A}=0.5$;

$\mathrm{dCorrB}=1$;

$\%$ Overall reaction enthalpy

$\mathrm{dHovr}=-20 \mathrm{e} 3 ; \% \mathrm{~J} / \mathrm{gmol}$

$\%$ BEP relationship parameters

alpha $=0.4$;

beta $=102 \mathrm{e} 3 ; \% \mathrm{~J} / \mathrm{gmol}$

$\%$ Input (1):

$\%$ Number of catalytic active sites (gmol)

$\mathrm{N}=\mathrm{u}$;

$\%$ States (2):

$\%$ Concentration of A in CSTR (M)

$\mathrm{Ca}=\mathrm{x}(1,1)$;

$\%$ Concentration of B in CSTR (M)

$\mathrm{Cb}=\mathrm{x}(2,1)$;

$\%$ Number of $\mathrm{A}^{*}$ in CSTR (gmol)

Ca_star $=\mathrm{x}(3,1)$;

$\%$ Number of $\mathrm{B}^{*}$ in CSTR (gmol)

Cb_star $=\mathrm{x}(4,1)$;

$\%$ Parameters:

$\%$ Binding energy $(\mathrm{J} / \mathrm{gmol})$

$\mathrm{BEa}=\mathrm{BEA} 0+\mathrm{dCorrA} *$ deltE0;

$\mathrm{BEb}=\mathrm{BEB} 0+\mathrm{dCorrB} * \operatorname{deltE} 0$;

$\%$ Heat of reaction $(\mathrm{J} / \mathrm{gmol})$

delH1 = -BEa; \% A --> A*

delH2 = dHovr + BEa $-\mathrm{BEb} ; \% \mathrm{~A}^{*}$--> B*

$\operatorname{delH} 3=\mathrm{BEb} ; \% \mathrm{~B}^{*}{ }_{-->} \mathrm{B}$

$\% \mathrm{E}$ - Activation energy in the Arrhenius Equation (J/gmol)

$\mathrm{R}=8.314459848 ; \% \mathrm{~J} / \mathrm{gmol}-\mathrm{K}$ 


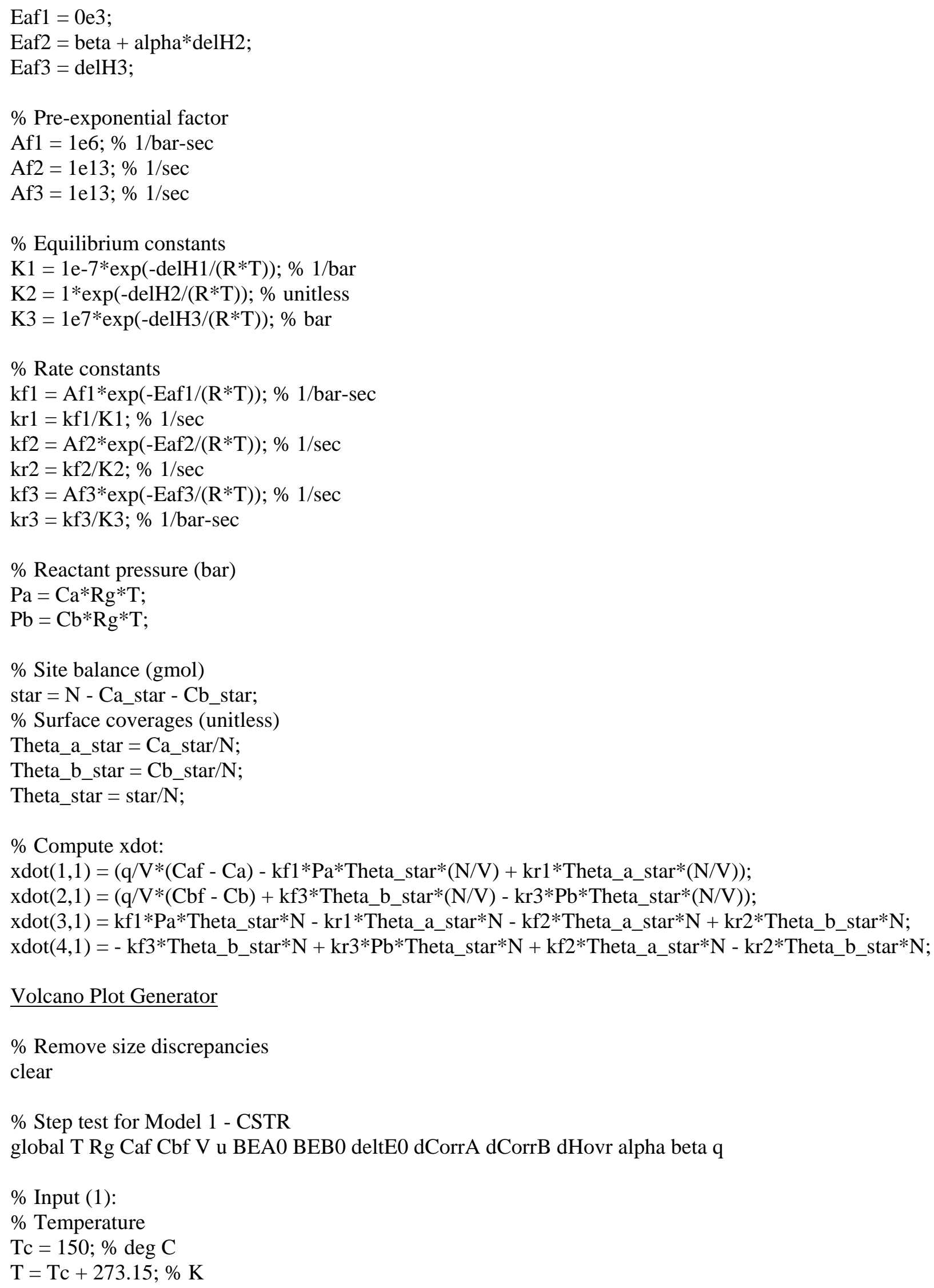




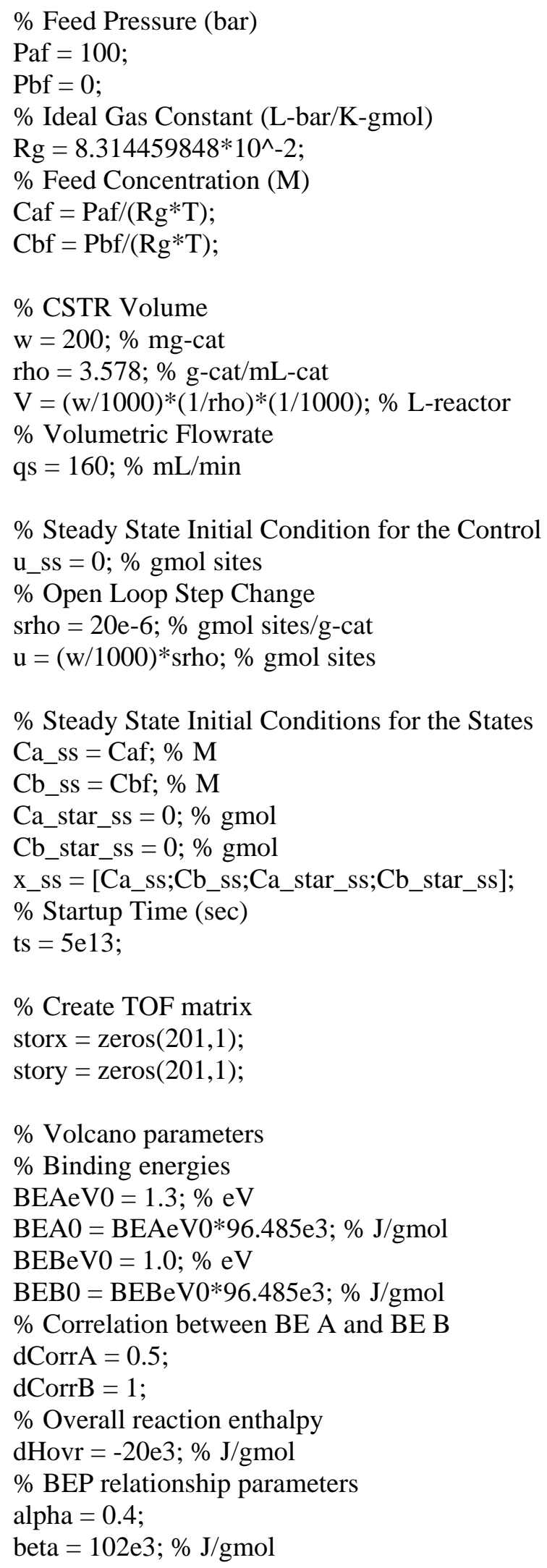




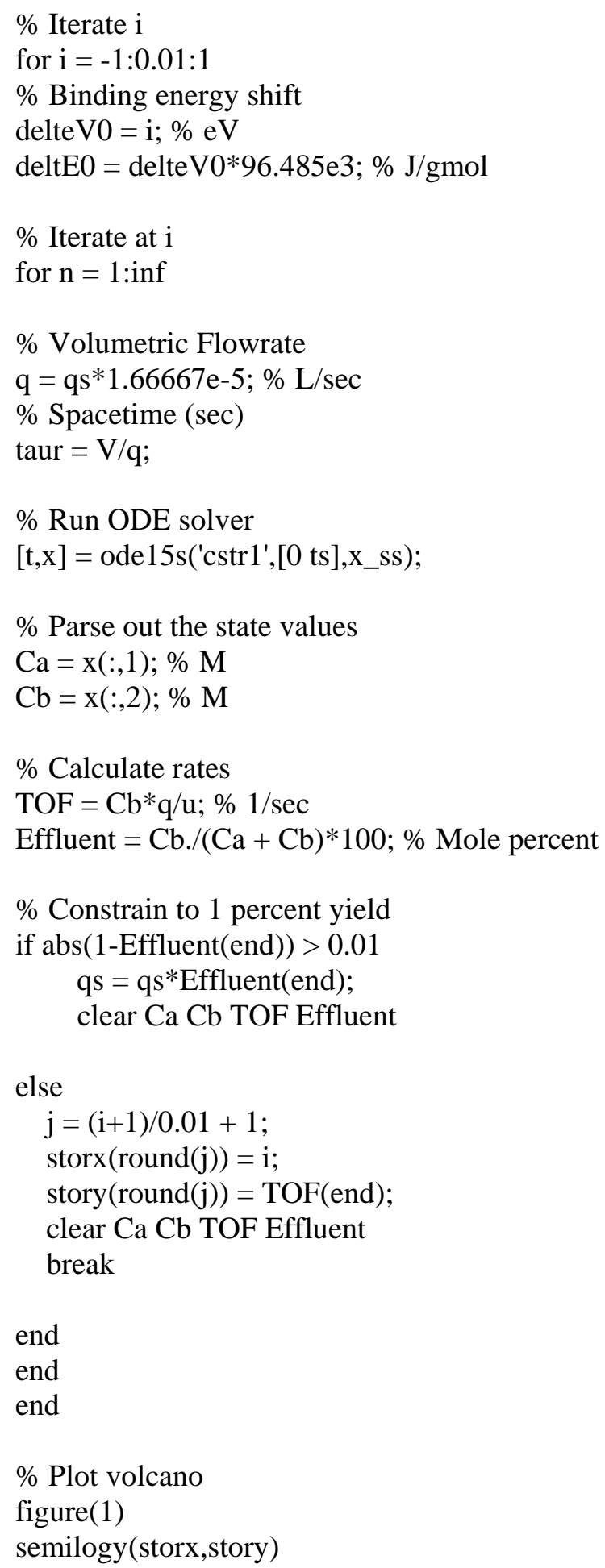

Dynamic Catalysis with Square, Triangle, or Sawtooth Waveforms

$\%$ Remove size discrepancies

clear

\% Step test for Models 1, 2, and 3 - CSTRs 


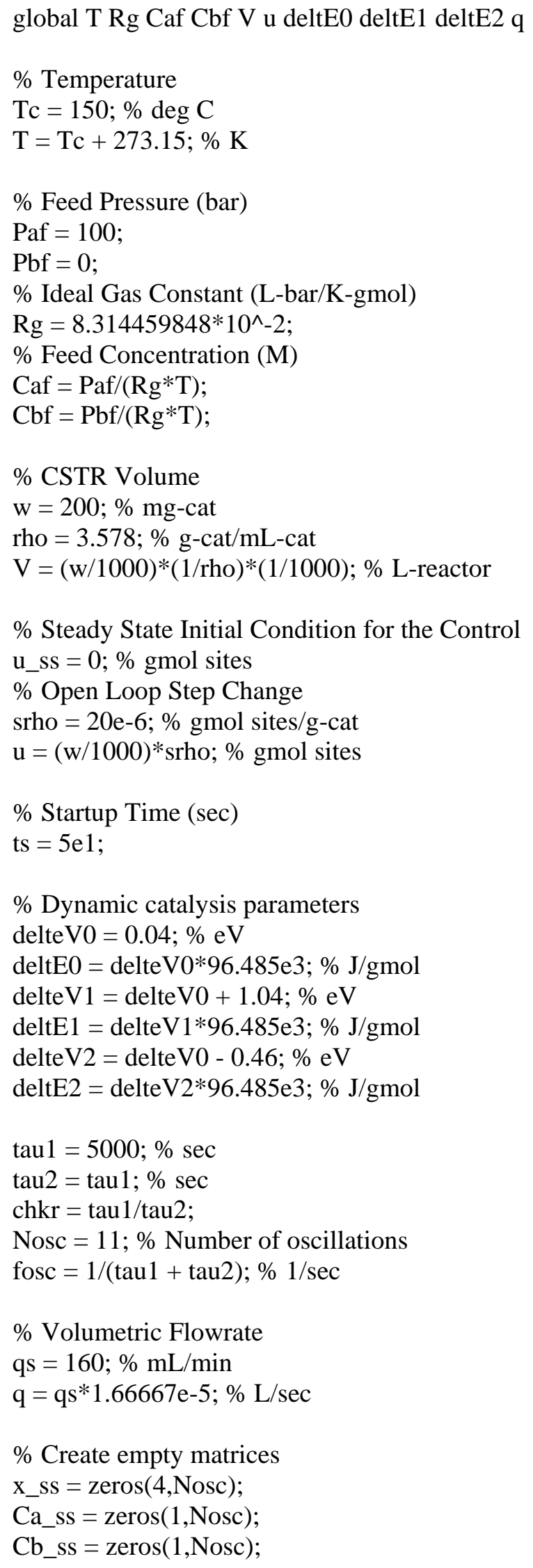


Ca_star_ss = zeros $(1$, Nosc $)$;

Cb_star_ss $=$ zeros $(1$, Nosc $)$;

$\%$ Steady State Initial Conditions for the States

Ca_ss(1) = Caf; \% M

Cb_ss $(1)=\mathrm{Cbf} ; \% \mathrm{M}$

Ca_star_ss $(1)=0 ; \%$ gmol

Cb_star_ss $(1)=0 ; \%$ gmol

X_ss(:,1) $=\left[\right.$ Ca_ss $\left.(1) ; C b \_s s(1) ; C a \_s t a r \_s s(1) ; C b \_s t a r \_s s(1)\right]$;

$\%$ Run ODE Solver

$[\mathrm{t}, \mathrm{x}]=$ ode15s('cstr1',[0 ts $\left.], \mathrm{x} \_s s(:, 1)\right)$;

$\%$ Parse out the state values

$\mathrm{Ca}(:, 1)=\mathrm{x}(:, 1) ; \% \mathrm{M}$

$\mathrm{Cb}(:, 1)=\mathrm{x}(:, 2) ; \% \mathrm{M}$

Ca_star(:,1) $=\mathrm{x}(:, 3) ; \%$ gmol

Cb_star(:,1) $=x(:, 4) ; \%$ gmol

star $=$ u - Ca_star(:,1) - Cb_star(:,1); \% gmol vacant sites

$\%$ Calculate rates

$\mathrm{TOF}=\operatorname{zeros}(\operatorname{size}(\mathrm{Cb}, 1)$, Nosc $)$;

$\operatorname{TOF}(:, 1)=\mathrm{Cb}(:, 1) * \mathrm{q} / \mathrm{u} ; \% 1 / \mathrm{sec}$

Effluent $=$ zeros $(\operatorname{size}(\mathrm{Cb}, 1)$, Nosc $)$;

Effluent $(:, 1)=\mathrm{Cb}(:, 1) . /(\mathrm{Ca}(:, 1)+\mathrm{Cb}(:, 1)) * 100 ; \%$ Mole percent

$\%$ Surface coverage (unitless)

Theta_a_star(:,1) = Ca_star(:,1)/u;

Theta_b_star(:,1) = Cb_star(:,1)/u;

Theta_star(:,1) $=\operatorname{star}(:, 1) / \mathrm{u}$;

$\%$ Create time matrix

tshift $=$ zeros $(\operatorname{size}(t, 1)$, Nosc $)$;

$\operatorname{tshift}(:, 1)=\mathrm{t}$;

$\%$ Create temporary storage

store $=$ zeros $($ Nosc, 5$)$;

$\operatorname{store}(1,:)=[\mathrm{x}(\mathrm{end},:), \mathrm{t}(\mathrm{end})]$;

$\%$ Begin dynamic catalysis

for $\mathrm{k}=2:$ Nosc

$\%$ Steady State Initial Conditions for the States

Ca_ss $(\mathrm{k})=\operatorname{store}(\mathrm{k}-1,1) ; \% \mathrm{M}$

Cb_ss $(\mathrm{k})=\operatorname{store}(\mathrm{k}-1,2) ; \% \mathrm{M}$

Ca_star_ss $(\mathrm{k})=\operatorname{store}(\mathrm{k}-1,3) ; \%$ gmol

Cb_star_ss $(\mathrm{k})=\operatorname{store}(\mathrm{k}-1,4) ; \%$ gmol

X_ss $(:, \mathrm{k})=\left[\mathrm{Ca} \_s s(\mathrm{k}) ; \mathrm{Cb} \_s s(\mathrm{k}) ; \mathrm{Ca} \_s t a r \_s s(\mathrm{k}) ; \mathrm{Cb} \_s t a r \_s s(\mathrm{k})\right]$;

\% CSTR Selection 


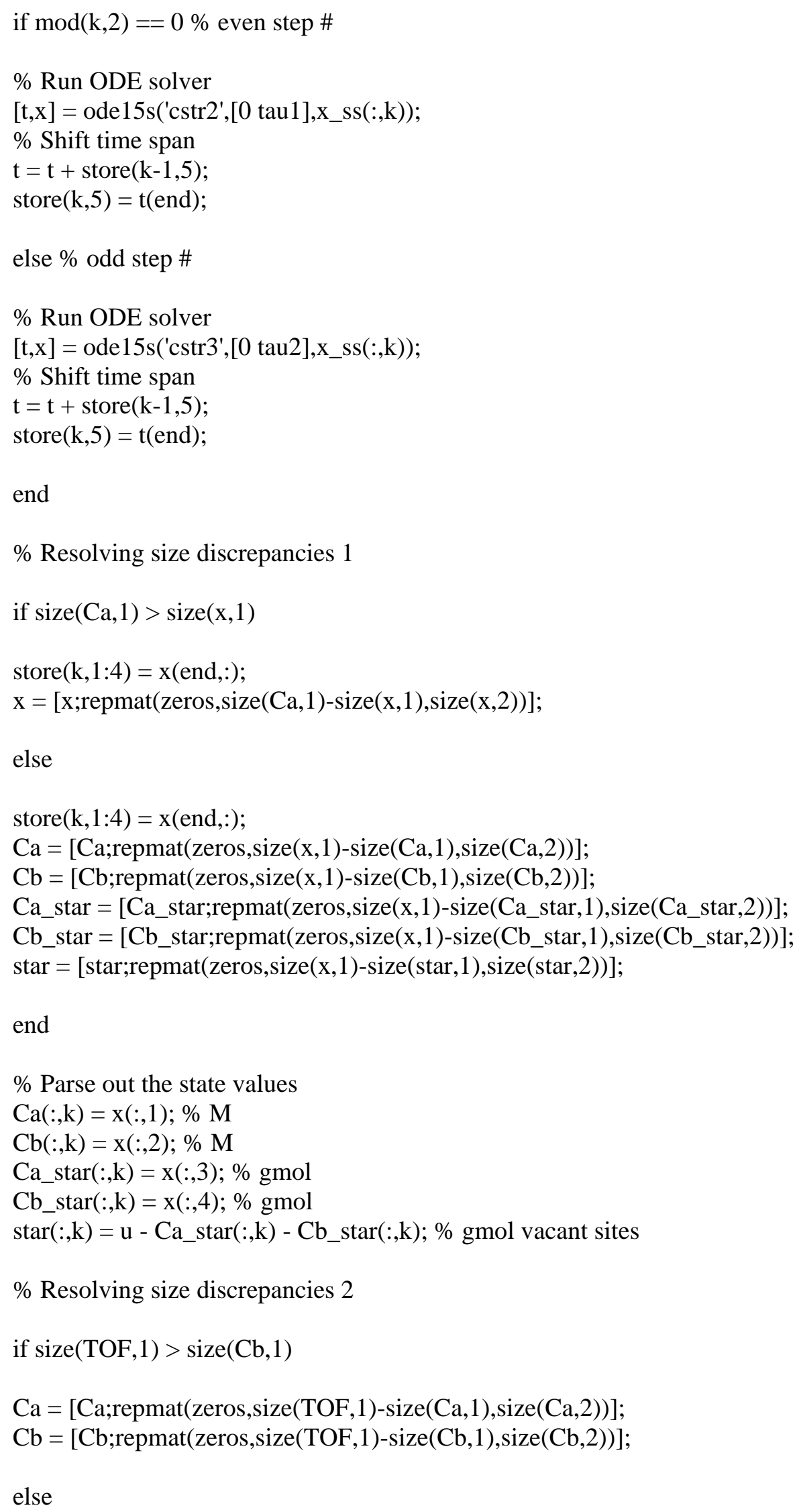


TOF $=[$ TOF;repmat (zeros,size $(\mathrm{Cb}, 1)-\operatorname{size}(\mathrm{TOF}, 1)$,size $(\mathrm{TOF}, 2))]$;

Effluent $=[$ Effluent;repmat $($ zeros,size $(\mathrm{Cb}, 1)-\operatorname{size}($ Effluent,1), size $($ Effluent,2)) $]$;

end

$\%$ Calculate rates

$\mathrm{TOF}(:, \mathrm{k})=\mathrm{Cb}(:, \mathrm{k}) * \mathrm{q} / \mathrm{u} ; \% 1 / \mathrm{sec}$

Effluent $(:, \mathrm{k})=\mathrm{Cb}(:, \mathrm{k}) . /(\mathrm{Ca}(:, \mathrm{k})+\mathrm{Cb}(:, \mathrm{k})) * 100 ; \%$ Mole percent

$\%$ Resolving size discrepancies 3

if $\operatorname{size}($ Theta_a_star,1) $>\operatorname{size}($ Ca_star,1)

Ca_star $=\left[\right.$ Ca_star;repmat $\left.\left(z e r o s, \operatorname{size}\left(T h e t a \_a \_s t a r, 1\right)-\operatorname{size}\left(C a \_s t a r, 1\right), \operatorname{size}\left(C a \_s t a r, 2\right)\right)\right]$;

Cb_star $=\left[\right.$ Cb_star;repmat $\left(z e r o s, \operatorname{size}\left(\right.\right.$ Theta_a_star,1)-size $\left.\left.\left(C b \_s t a r, 1\right), \operatorname{size}\left(C b \_s t a r, 2\right)\right)\right]$;

star $=[\operatorname{star} ; r$ repmat $(z e r o s$, size $($ Theta_a_star,1)-size $(\operatorname{star}, 1), \operatorname{size}(\operatorname{star}, 2))]$;

else

Theta_a_star $=\left[\right.$ Theta_a_star;repmat $\left(z e r o s, \operatorname{size}\left(C a \_s t a r, 1\right)-\operatorname{size}(\right.$ Theta_a_star,1),size $($ Theta_a_star,2) $)]$;

Theta_b_star $=$ [Theta_b_star;repmat $\left(z e r o s, \operatorname{size}\left(C a \_s t a r, 1\right)-\operatorname{size}(\right.$ Theta_b_star,1),size $($ Theta_b_star,2))];

Theta_star $=\left[\right.$ Theta_star;repmat $\left(z e r o s, s i z e\left(C a \_s t a r, 1\right)-\operatorname{size}(\right.$ Theta_star,1),size $($ Theta_star,2))];

end

$\%$ Surface coverage (unitless)

Theta_a_star(:,k) = Ca_star(:,k)/u;

Theta_b_star(:,k) $=$ Cb_star(:,k)/u;

Theta_star(:,k) = star(:,k)/u;

$\%$ Correcting time matrix

$\%$ Resolving size discrepancies 4

if size (tshift, 1$)>\operatorname{size}(\mathrm{t}, 1)$

$\mathrm{t}=[\mathrm{t} ; \operatorname{repmat}($ zeros, size $(\mathrm{tshift}, 1)-\operatorname{size}(\mathrm{t}, 1), \operatorname{size}(\mathrm{t}, 2))]$;

else

tshift $=[$ tshift;repmat $($ zeros, size $(t, 1)-\operatorname{size}(\operatorname{tshift}, 1), \operatorname{size}(t s h i f t, 2))]$

end

$\operatorname{tshift}(:, \mathrm{k})=\mathrm{t}$;

end

$\%$ End dynamic catalysis

$\%$ Clean up data

Page S9 


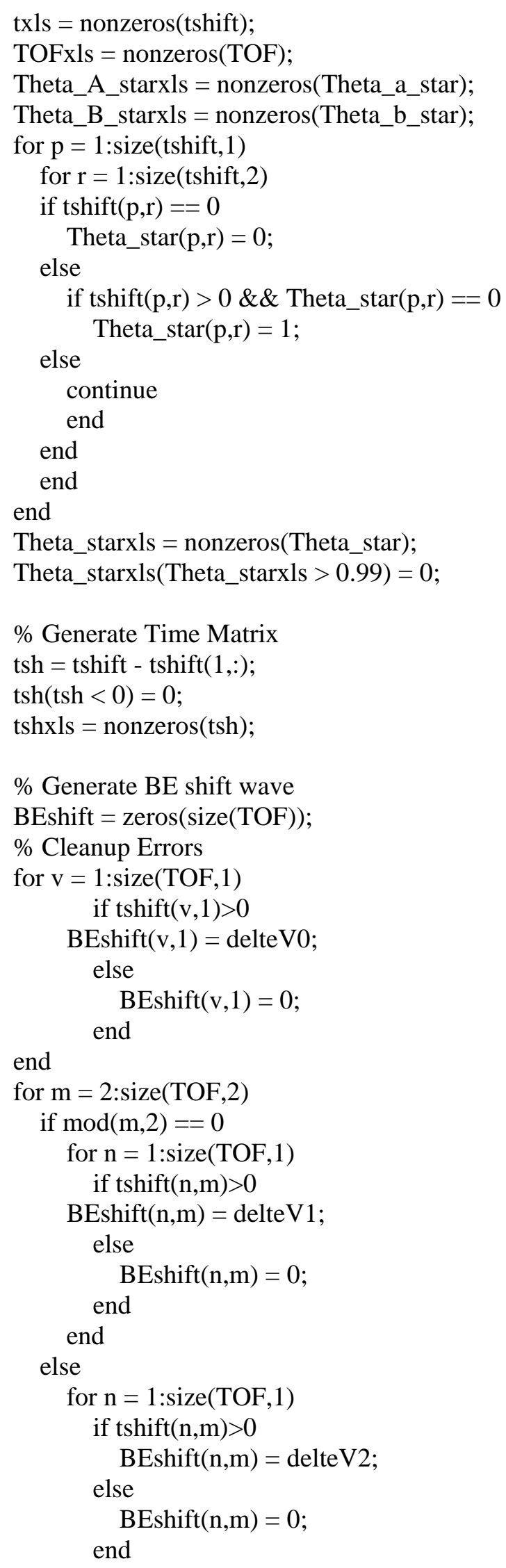




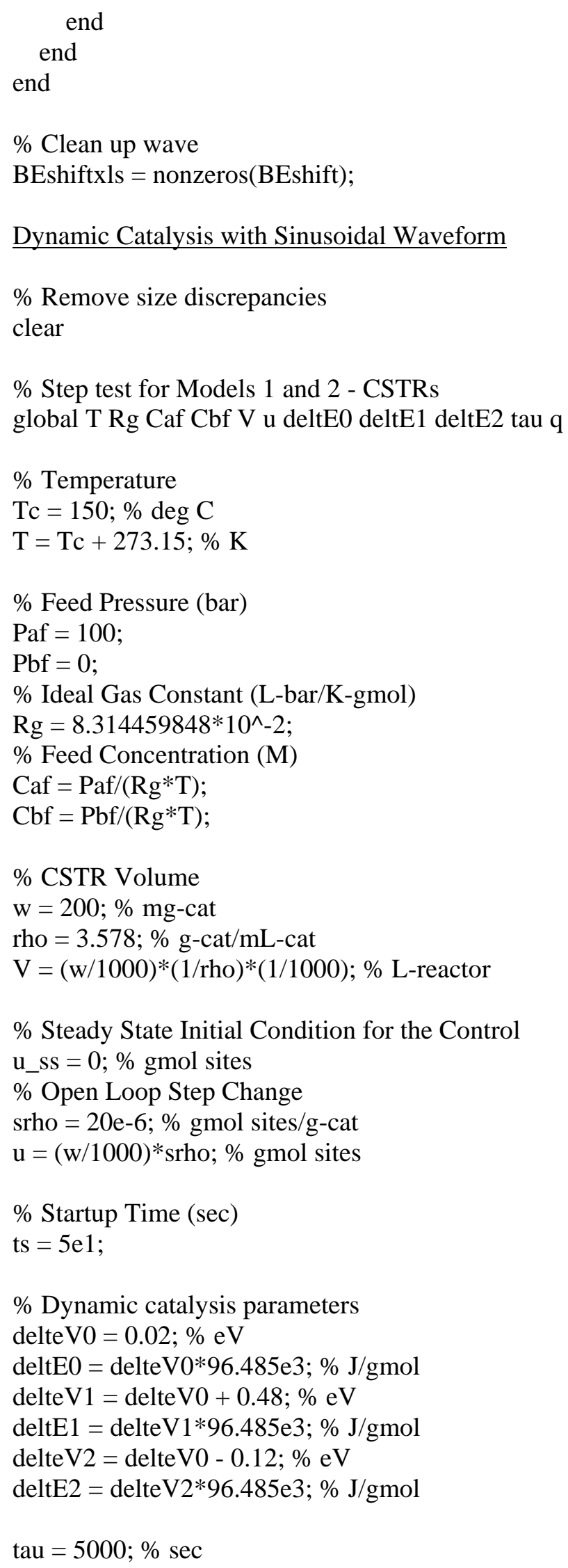




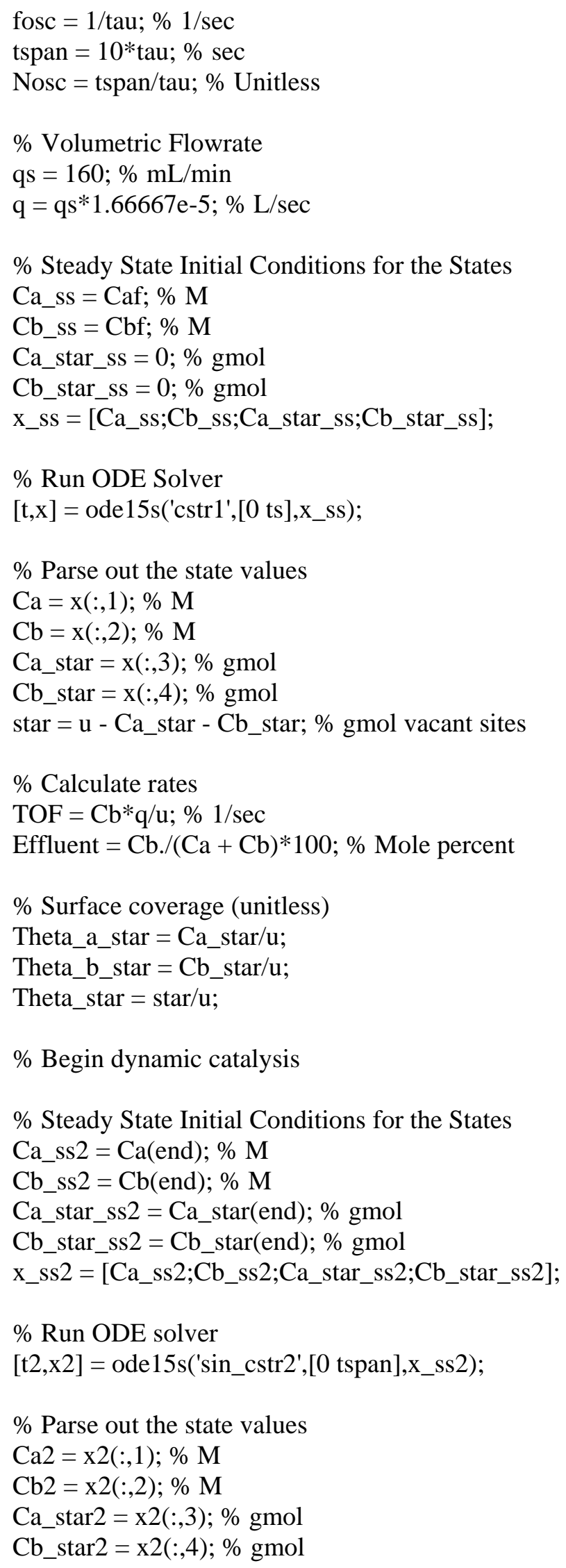

$\%$ Parse out the state values

$\mathrm{Ca} 2=\mathrm{x} 2(:, 1) ; \% \mathrm{M}$

$\mathrm{Cb} 2=\mathrm{x} 2(:, 2) ; \% \mathrm{M}$

Ca_star2 $=x 2(:, 3) ; \%$ gmol

Cb_star2 $=x 2(:, 4) ; \%$ gmol 
star2 = u - Ca_star2 - Cb_star2; \% gmol vacant sites

$\%$ Calculate rates

$\mathrm{TOF} 2=\mathrm{Cb} 2 * \mathrm{q} / \mathrm{u} ; \% 1 / \mathrm{sec}$

Effluent $2=\mathrm{Cb} 2 . /(\mathrm{Ca} 2+\mathrm{Cb} 2) * 100 ; \%$ Mole percent

$\%$ Surface coverage (unitless)

Theta_a_star $2=$ Ca_star $2 / \mathrm{u}$;

Theta_b_star2 $=$ Cb_star $2 / \mathrm{u}$;

Theta_star2 = star2/u;

$\%$ Shift time matrix

$\mathrm{t} 2 \mathrm{~s}=\mathrm{t} 2+\mathrm{t}($ end $)$

$\%$ End dynamic catalysis

$\%$ Clean up data

txls $=[$ nonzeros $(\mathrm{t}) ;$ nonzeros $(\mathrm{t} 2 \mathrm{~s})]$;

TOFxls = [nonzeros(TOF);nonzeros(TOF2)];

Theta_A_starxls $=[$ nonzeros(Theta_a_star);nonzeros(Theta_a_star2) $]$;

Theta_B_starxls $=$ [nonzeros(Theta_b_star);nonzeros(Theta_b_star2)];

Theta_starxls $=$ [nonzeros(Theta_star);nonzeros(Theta_star2)];

$\%$ Generate BE shift wave

BEshift $1=\operatorname{zeros}(\operatorname{size}(\mathrm{t}))$;

BEshift1(2:end,1) = delteV0;

BEshift2 = ((delteV2-delteV1)/2 $) * \cos (2 *$ pi $*$ t2/tau $)+$ mean $([$ delteV1, delteV2] $)$;

BEshiftxls $=[$ nonzeros $($ BEshift1);nonzeros $($ BEshift2 $)]$; 


\section{Section S2. ODE Solver Selection and Justification}

Matlab ODE solvers were screened using a sinusoidal dynamic catalysis waveform. ODE45 is the recommended solver for general use in Matlab, however, this solver failed to generate a solution after $8 \mathrm{~h}$ of continuous computation.

Stats for ODE15s:

3179 successful steps

312 failed attempts

4727 function evaluations

1 partial derivatives

631 LU decompositions

4721 solutions of linear systems

Elapsed time is 1.449973 seconds.

Stats for ODE23s:

10561 successful steps

44 failed attempts

74017 function evaluations

10561 partial derivatives

10605 LU decompositions

31815 solutions of linear systems

Elapsed time is 10.730825 seconds.

Stats for ODE23t:

14214 successful steps

125 failed attempts

21663 function evaluations

45 partial derivatives

1015 LU decompositions

21437 solutions of linear systems

Elapsed time is 4.710374 seconds.

Stats for ODE23tb:

11161 successful steps

92 failed attempts

30658 function evaluations

5 partial derivatives

897 LU decompositions

41881 solutions of linear systems

Elapsed time is 4.704730 seconds.

ODE23s has the least number of failed attempts, but ODE15s performed most efficiently in terms of number of steps and amount of time to generate the solution. Therefore, ODE15s was used throughout this manuscript to solve CSTR equations under static and dynamic catalysis conditions. 
Section S3. Time on Stream Data for Static Catalysis
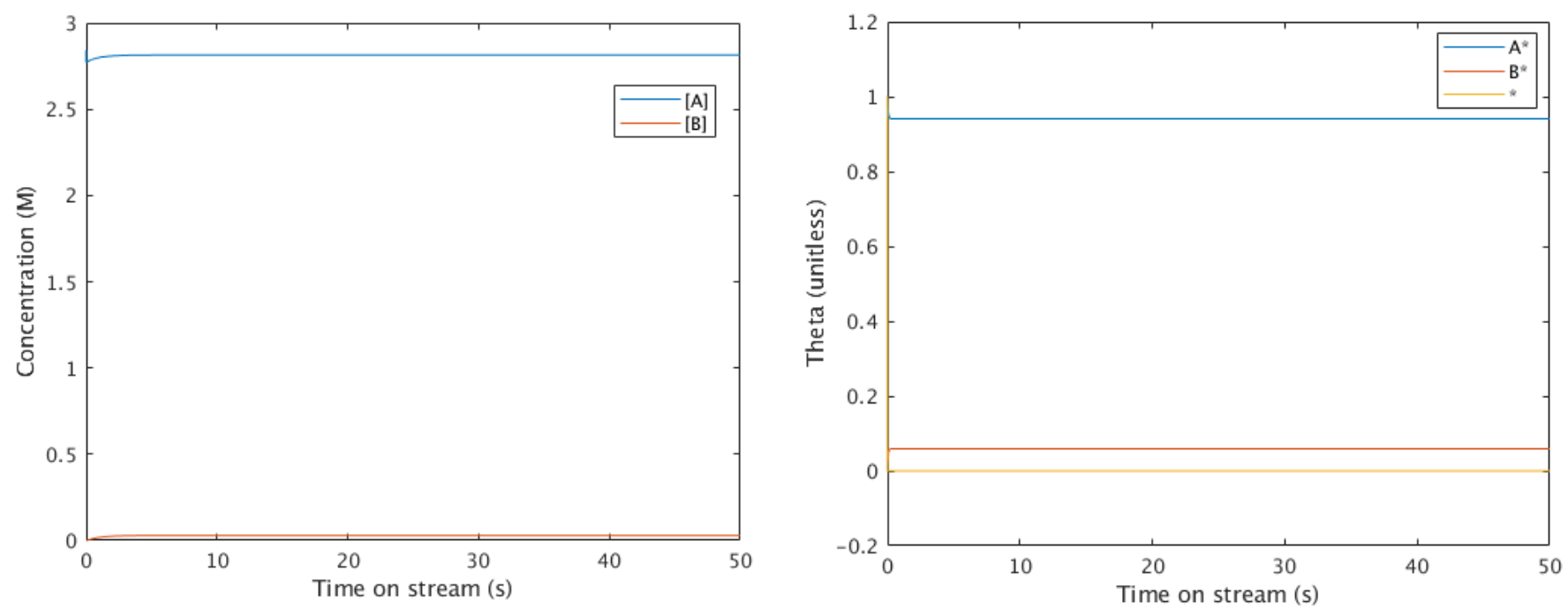

Figure S1. Example of time on stream data generated with ODE15s for a CSTR operating at $1 \%$ yield of B. Reaction conditions: $150{ }^{\circ} \mathrm{C}, 100$ bar A inlet, and a volumetric spacetime of $1 \mathrm{~s}$. 


\section{Section S4. Time on Stream Data for Dynamic Catalysis}

Section S4.1. Varying Oscillation Frequency with a Square Waveform
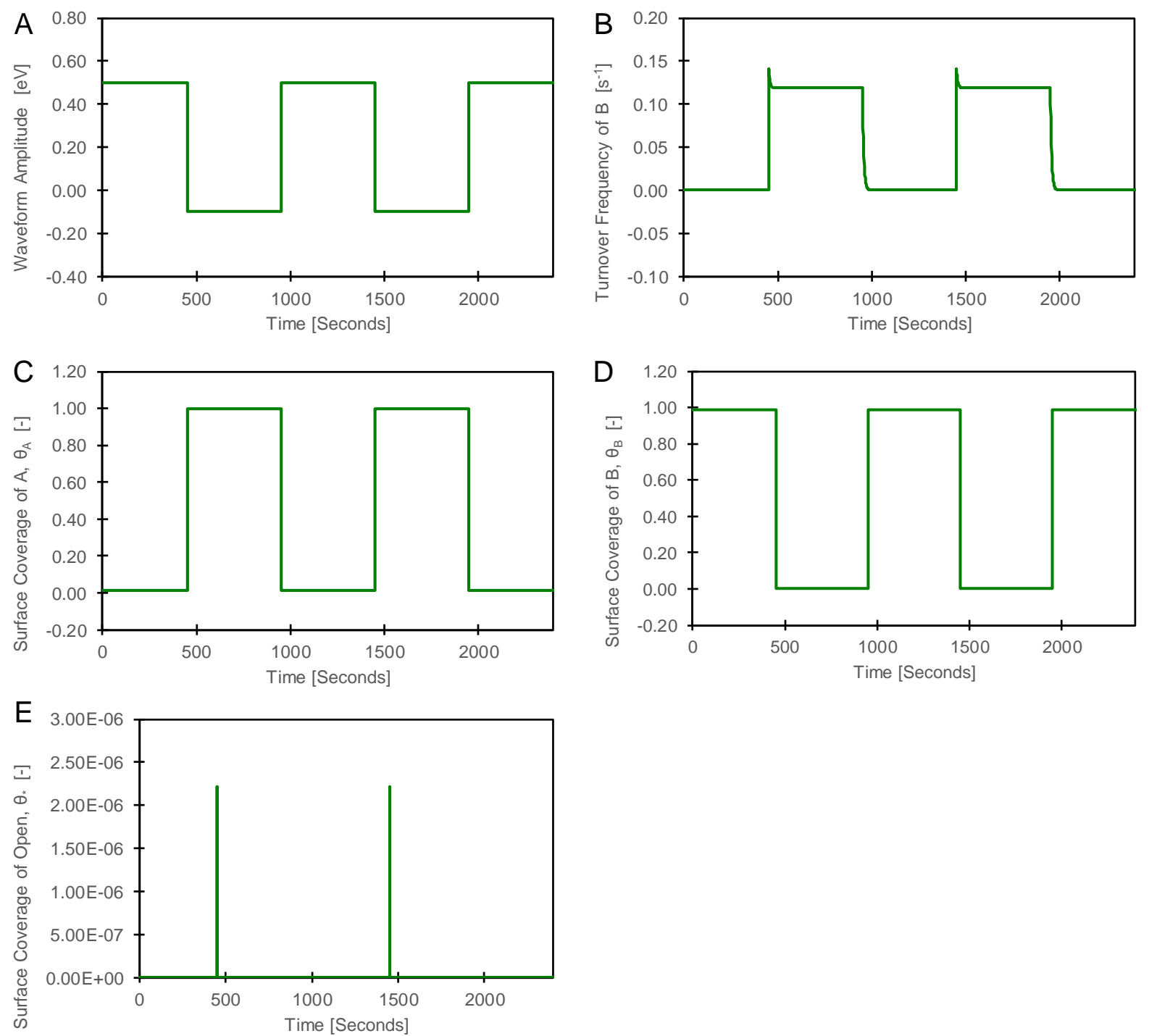

Figure S2. Dynamics of $\mathbf{0 . 0 0 1} \mathbf{~ H z}$ System. A. Waveform amplitude of -0.1 to $+0.5 \mathrm{eV}$. B. Catalytic turnover frequency of B with time. C. Surface coverage of A* with time. D. Surface coverage of B* with time. E. Surface coverage of open sites with time. Conditions: $150{ }^{\circ} \mathrm{C}, 100$ bar A inlet, $\Delta \mathrm{U}=0.6 \mathrm{eV}, 1 \%$ yield of B, CSTR reactor, oscillation frequency $0.001 \mathrm{~Hz}$, Volcano Curve Parameters: $\alpha$ of 0.8 and $\mathrm{E}_{0}$ of $102 \mathrm{~kJ} / \mathrm{mol}, \mathrm{B}^{*}$ shifts twice as much as $\mathrm{A}^{*}$ 

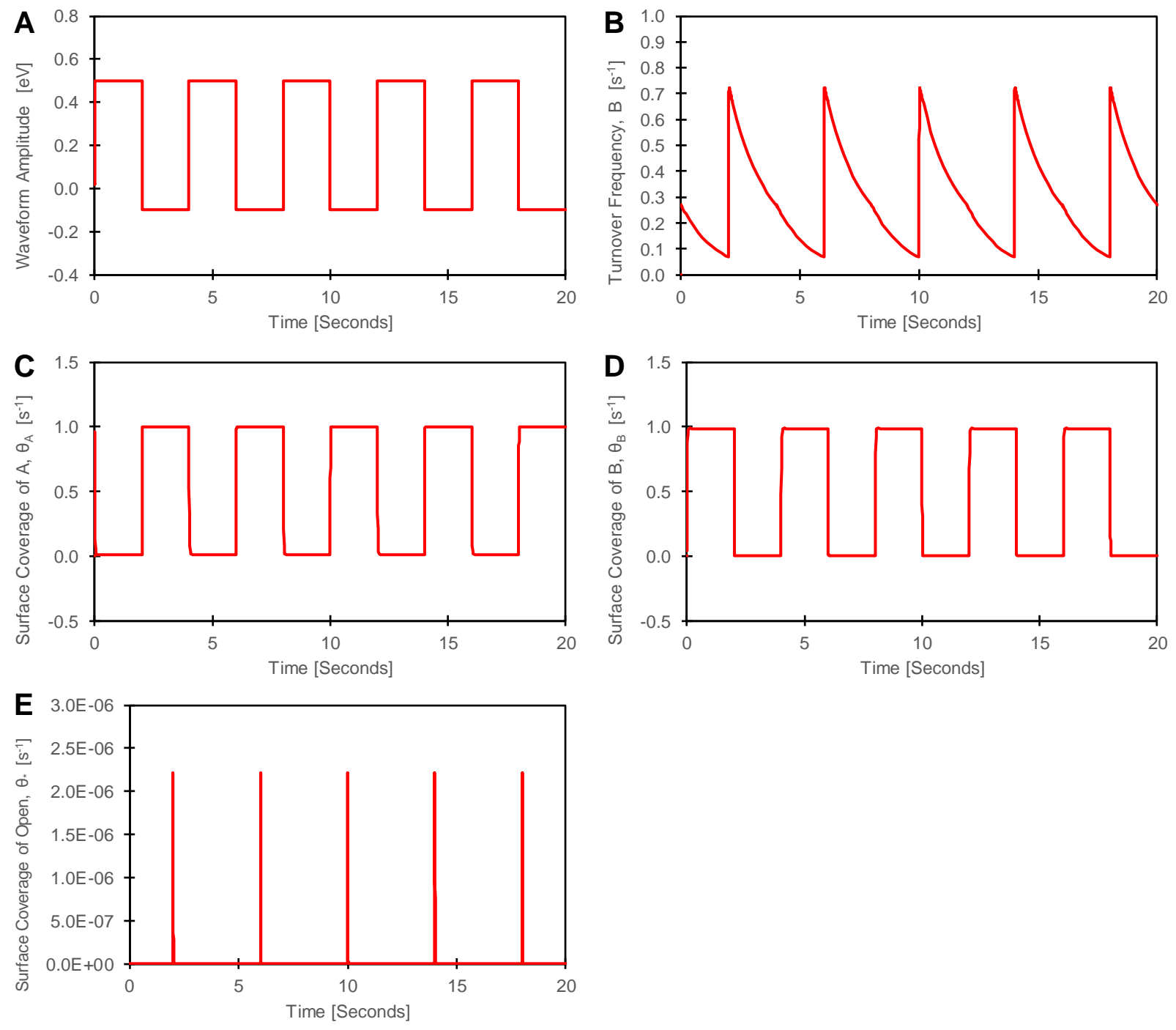

Figure S3. Dynamics of $0.25 \mathrm{~Hz}$ System. A. Waveform amplitude of -0.1 to $+0.5 \mathrm{eV}$. B. Catalytic turnover frequency of B with time. C. Surface coverage of A* with time. D. Surface coverage of B* with time. E. Surface coverage of open sites with time. Conditions: $150{ }^{\circ} \mathrm{C}, 100$ bar A inlet, $\Delta \mathrm{U}=0.6 \mathrm{eV}, 1 \%$ yield of B, CSTR reactor, oscillation frequency $0.25 \mathrm{~Hz}$, Volcano Curve Parameters: $\alpha$ of 0.8 and $\mathrm{E}_{0}$ of $102 \mathrm{~kJ} / \mathrm{mol}, \mathrm{B}^{*}$ shifts twice as much as $\mathrm{A}^{*}$ 

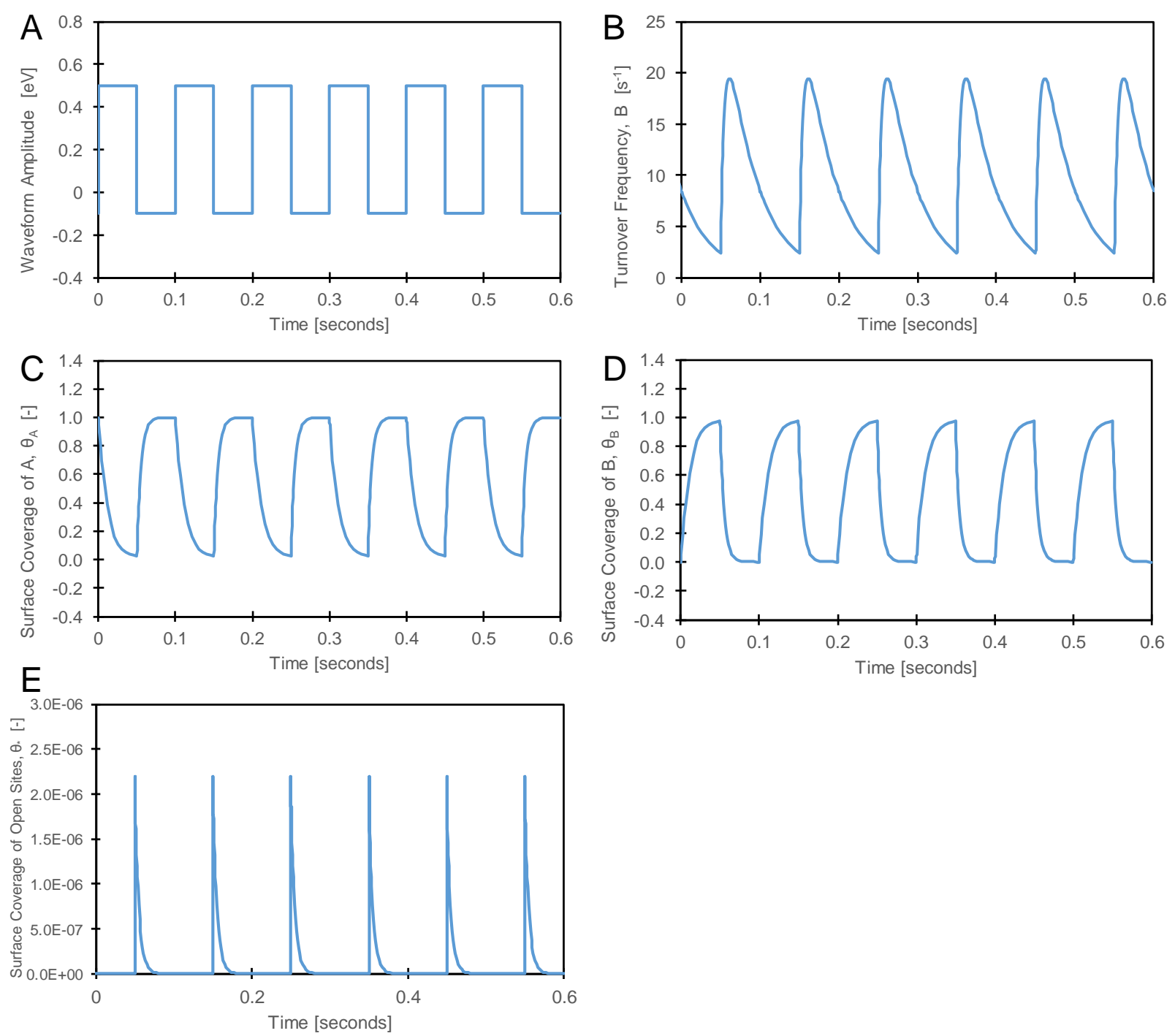

Figure S4. Dynamics of $10 \mathrm{~Hz}$ System. A. Waveform amplitude of -0.1 to $+0.5 \mathrm{eV}$. B. Catalytic turnover frequency of B with time. C. Surface coverage of A* with time. D. Surface coverage of B* with time. E. Surface coverage of open sites with time. Conditions: $150{ }^{\circ} \mathrm{C}, 100$ bar A inlet, $\Delta \mathrm{U}=0.6 \mathrm{eV}, 1 \%$ yield of B, CSTR reactor, oscillation frequency $10 \mathrm{~Hz}$, Volcano Curve Parameters: $\alpha$ of 0.8 and $\mathrm{E}_{0}$ of $102 \mathrm{~kJ} / \mathrm{mol}, \mathrm{B}^{*}$ shifts twice as much as $\mathrm{A}^{*}$ 

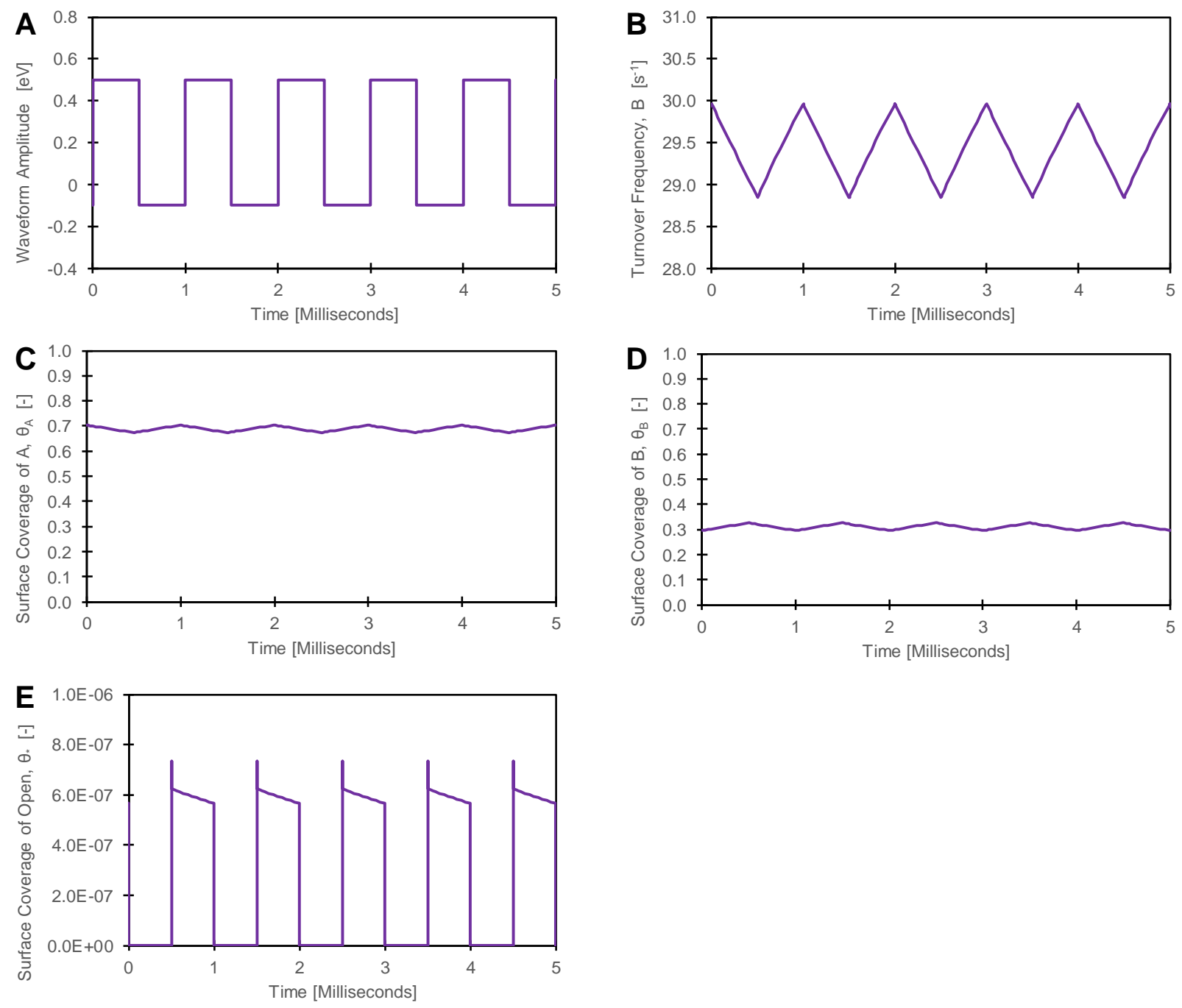

Figure S5. Dynamics of $\mathbf{1 , 0 0 0 ~ H z ~ S y s t e m . ~ A . ~ W a v e f o r m ~ a m p l i t u d e ~ o f ~}-0.1$ to $+0.5 \mathrm{eV}$. B. Catalytic turnover frequency of B with time. C. Surface coverage of A* with time. D. Surface coverage of B* with time. E. Surface coverage of open sites with time. Conditions: $150{ }^{\circ} \mathrm{C}, 100$ bar inlet $\mathrm{A}, \Delta \mathrm{U}=0.6 \mathrm{eV}$, $1 \%$ yield of B, CSTR reactor, oscillation frequency $1,000 \mathrm{~Hz}$, Volcano Curve Parameters: $\alpha$ of 0.8 and $\mathrm{E}_{0}$ of $102 \mathrm{~kJ} / \mathrm{mol}, \mathrm{B}^{*}$ shifts twice as much as $\mathrm{A}^{*}$ 
$\underline{\text { Section S4.2. } 10 \mathrm{~Hz} \text { Dynamic Catalysis with Varying Waveforms }}$
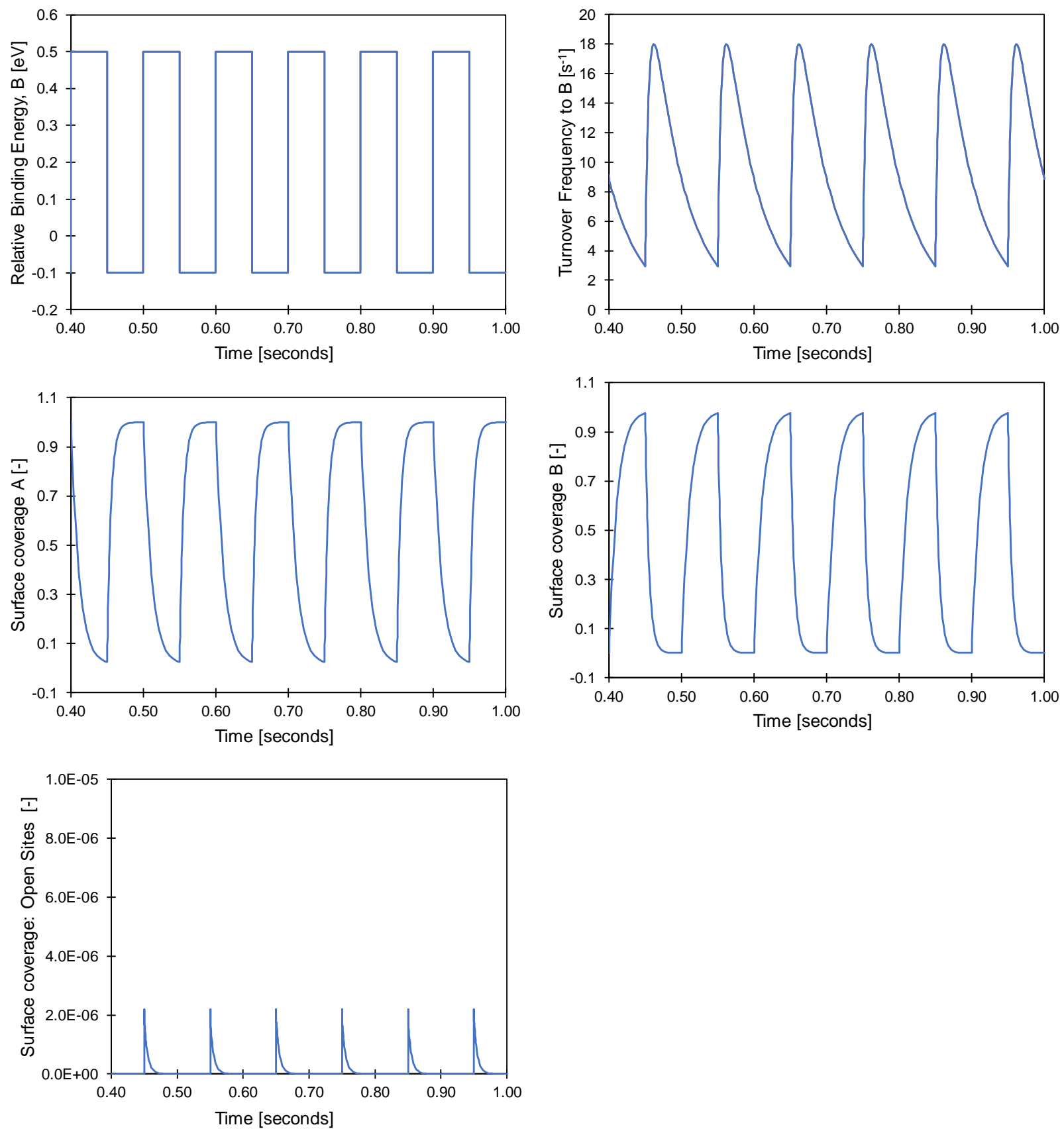

Figure S6. Square waveform at $10 \mathrm{~Hz}$. Conditions: A CSTR reactor for A converting to B operating at $1 \%$ yield, $150{ }^{\circ} \mathrm{C}$, and 100 bar A inlet. Volcano plot: $\Delta \mathrm{U} \sim 0.6 \mathrm{eV}, \alpha \sim 0.8, \mathrm{E}_{0}$ of $102 \mathrm{~kJ} / \mathrm{mol}$, and B* moves twice as much as $\mathrm{A}^{*}$ 

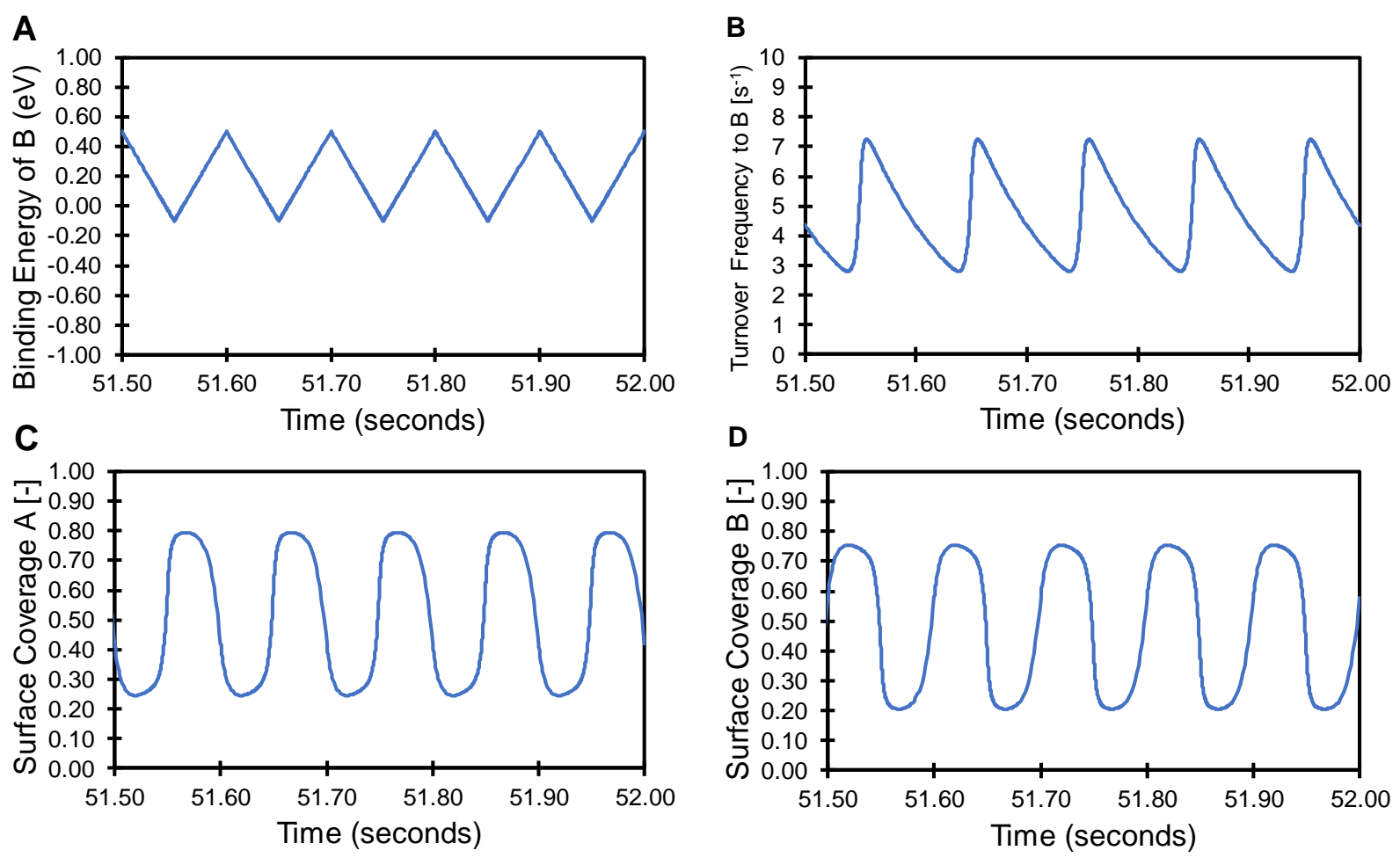

Figure S7. Triangle waveform at $10 \mathrm{~Hz}$. Conditions: A CSTR reactor for A converting to B operating at $1 \%$ yield, $150{ }^{\circ} \mathrm{C}$, and 100 bar A inlet. Volcano plot: $\Delta \mathrm{U} \sim 0.6 \mathrm{eV}, \alpha \sim 0.8, \mathrm{E}_{0}$ of $102 \mathrm{~kJ} / \mathrm{mol}$, and B* moves twice as much as $\mathrm{A}^{*}$ 

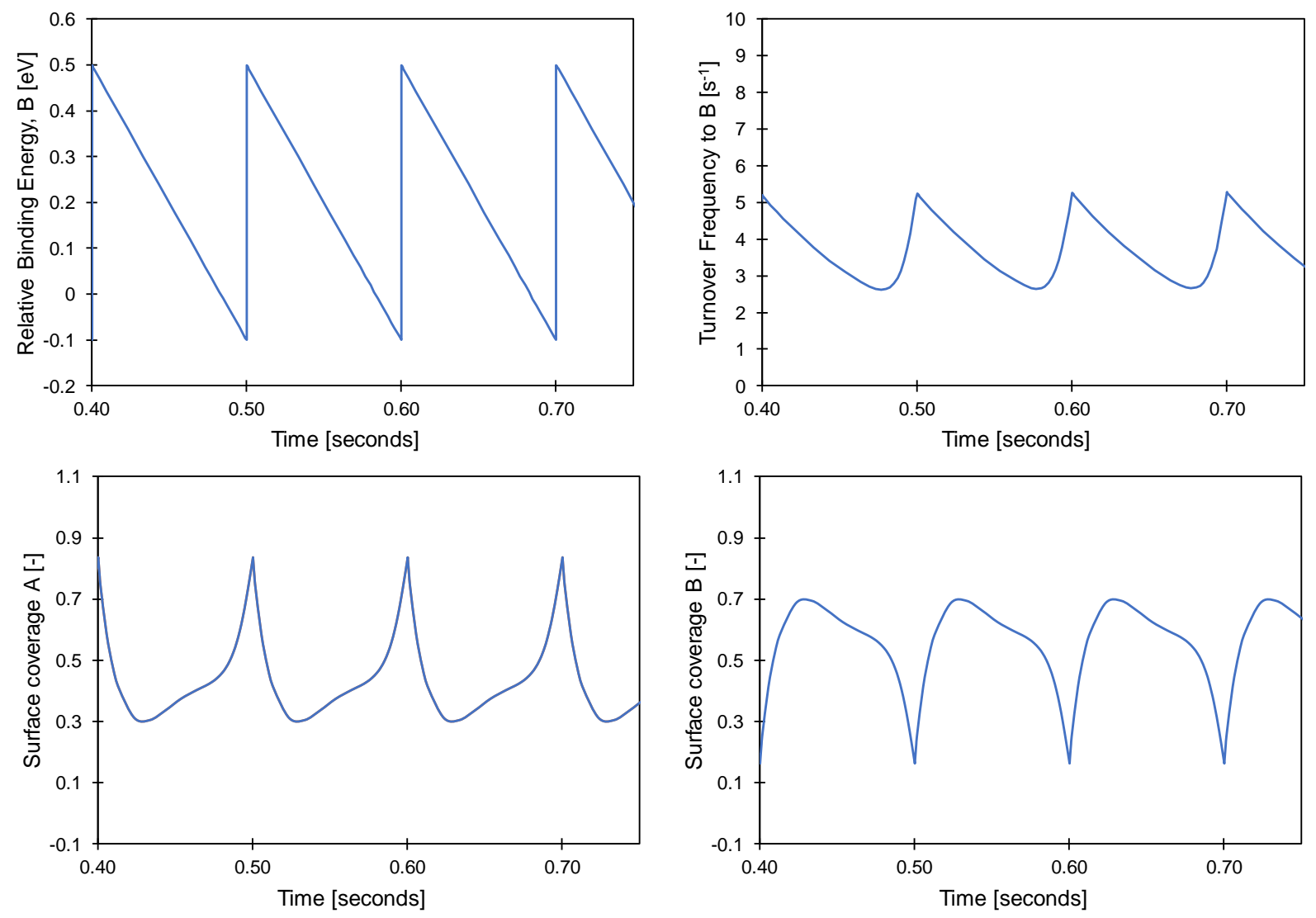

Figure S8. Sawtooth waveform at $10 \mathrm{~Hz}$. Conditions: A CSTR reactor for A converting to B operating at $1 \%$ yield, $150{ }^{\circ} \mathrm{C}$, and 100 bar inlet $\mathrm{A}$. Volcano plot: $\Delta \mathrm{U} \sim 0.6 \mathrm{eV}, \alpha \sim 0.8, \mathrm{E}_{0}$ of $102 \mathrm{~kJ} / \mathrm{mol}$, and $\mathrm{B}^{*}$ moves twice as much as $\mathrm{A}^{*}$ 

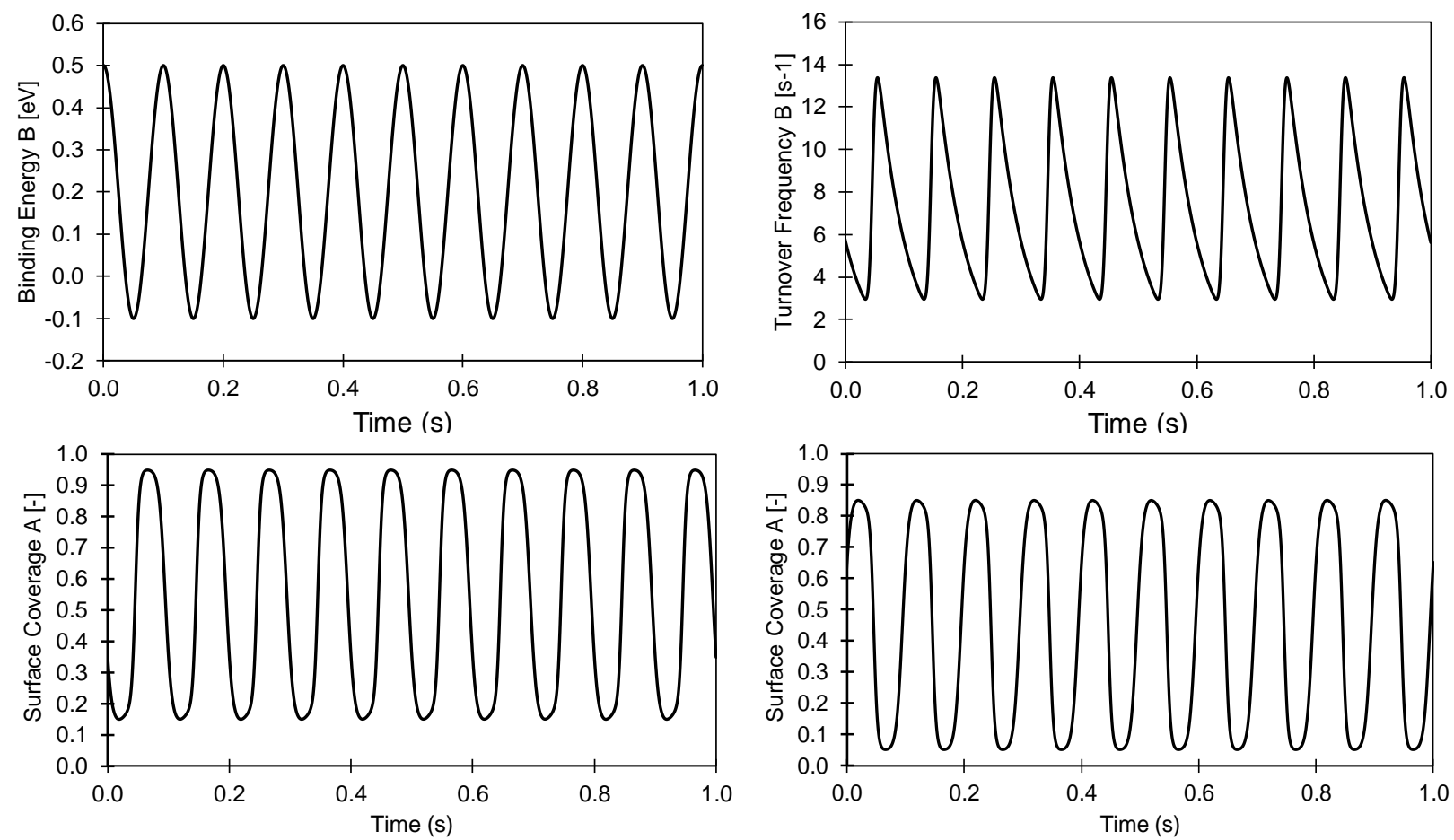

Figure S9. Sinusoidal waveform at $10 \mathrm{~Hz}$. Conditions: A CSTR reactor for A converting to B operating at $1 \%$ yield, $150{ }^{\circ} \mathrm{C}$, and 100 bar inlet $\mathrm{A}$. Volcano plot: $\Delta \mathrm{U} \sim 0.6 \mathrm{eV}, \alpha \sim 0.8, \mathrm{E}_{0}$ of $102 \mathrm{~kJ} / \mathrm{mol}$, and B* moves twice as much as $\mathrm{A}^{*}$ 


\section{Section S5. Figure 3 Heatmap Data}

Table S1. Heatmap data (TOFs [=] 1/s) for oscillation amplitudes between $0.0-1.0 \mathrm{eV}$ and frequencies between $10^{-4}-10^{10} \mathrm{~Hz}$. Volcano parameters: $\alpha$ of $0.8, \mathrm{E}_{0}$ of $102 \mathrm{~kJ} / \mathrm{mol}, 150{ }^{\circ} \mathrm{C}, 100 \mathrm{bar}, 1 \%$ yield of product $\mathrm{B}$, and $\mathrm{B}^{*}$ shifts twice as much as $\mathrm{A}^{*}$

\begin{tabular}{l|ccccccccccc}
\multicolumn{1}{l}{} & $\mathbf{0 . 0} \mathbf{e V}$ & $\mathbf{0 . 1} \mathbf{e V}$ & $\mathbf{0 . 2} \mathbf{e V}$ & $\mathbf{0 . 3} \mathbf{~ e V}$ & $\mathbf{0 . 4} \mathbf{e V}$ & $\mathbf{0 . 5} \mathbf{~ e V}$ & $\mathbf{0 . 6} \mathbf{e V}$ & $\mathbf{0 . 7} \mathbf{~ e V}$ & $\mathbf{0 . 8} \mathbf{e V}$ & $\mathbf{0 . 9} \mathbf{e V}$ & $\mathbf{1 . 0} \mathbf{e V}$ \\
\cline { 2 - 12 } $\mathbf{1 0}^{-4} \mathbf{H z}$ & 0.2609 & 0.2106 & 0.1397 & 0.1219 & 0.1021 & 0.0779 & 0.0621 & 0.0465 & 0.0289 & 0.0216 & 0.0174 \\
$\mathbf{1 0}^{-3} \mathbf{H z}$ & 0.2609 & 0.2106 & 0.1442 & 0.1219 & 0.1021 & 0.0808 & 0.0621 & 0.0403 & 0.0289 & 0.0216 & 0.0181 \\
$\mathbf{1 0}^{-\mathbf{2}} \mathbf{H z}$ & 0.2609 & 0.2056 & 0.1407 & 0.1219 & 0.1021 & 0.0808 & 0.0617 & 0.0556 & 0.0364 & 0.0325 & 0.0293 \\
$\mathbf{1 0}^{-\mathbf{1}} \mathbf{H z}$ & 0.2609 & 0.2263 & 0.1989 & 0.1926 & 0.1907 & 0.1820 & 0.1695 & 0.1501 & 0.1334 & 0.1232 & 0.1113 \\
$\mathbf{1 0}^{\mathbf{0}} \mathbf{H z}$ & 0.2609 & 0.2972 & 0.4505 & 0.7468 & 0.8541 & 0.9143 & 1.001 & 1.139 & 1.288 & 1.541 & 2.366 \\
$\mathbf{1 0}^{\mathbf{1}} \mathbf{H z}$ & 0.2609 & 0.4149 & 0.9343 & 2.231 & 4.321 & 7.425 & 8.030 & 8.721 & 9.325 & 13.26 & 28.00 \\
$\mathbf{1 0}^{\mathbf{2}} \mathbf{H z}$ & 0.2609 & 0.4202 & 0.9620 & 2.371 & 5.581 & 12.81 & 26.88 & 49.68 & 90.94 & 120.1 & 152.9 \\
$\mathbf{1 0}^{\mathbf{3}} \mathbf{H z}$ & 0.2609 & 0.4203 & 0.9632 & 2.385 & 5.611 & 13.04 & 29.08 & 65.08 & 142.4 & 296.3 & 542.5 \\
$\mathbf{1 0}^{\mathbf{4}} \mathbf{H z}$ & 0.2609 & 0.4203 & 0.9634 & 2.383 & 5.618 & 13.05 & 29.43 & 65.38 & 144.0 & 315.9 & 690.5 \\
$\mathbf{1 0}^{\mathbf{5}} \mathbf{H z}$ & 0.2609 & 0.3990 & 0.9550 & 2.383 & 5.616 & 13.05 & 29.43 & 65.39 & 144.2 & 316.6 & 693.1 \\
$\mathbf{1 0}^{\mathbf{6}} \mathbf{H z}$ & 0.2609 & 0.2750 & 0.3364 & 0.8856 & 4.947 & 13.01 & 29.43 & 65.39 & 144.2 & 316.7 & 693.9 \\
$\mathbf{1 0}^{\mathbf{7}} \mathbf{H z}$ & 0.2609 & 0.2609 & 0.2613 & 0.2667 & 0.2833 & 0.3799 & 3.078 & 59.92 & 144.0 & 316.7 & 694.0 \\
$\mathbf{1 0}^{\mathbf{8}} \mathbf{H z}$ & 0.2609 & 0.2609 & 0.2609 & 0.2611 & 0.2617 & 0.2637 & 0.2700 & 0.2917 & 0.4478 & 17.81 & 40.14 \\
$\mathbf{1 0}^{\mathbf{9}} \mathbf{H z}$ & 0.2609 & 0.2609 & 0.2609 & 0.2609 & 0.2609 & 0.2611 & 0.2614 & 0.2622 & 0.2645 & 0.2719 & 0.3063 \\
$\mathbf{1 0}^{\mathbf{1 0}} \mathbf{H z}$ & 0.2609 & 0.2609 & 0.2609 & 0.2609 & 0.2609 & 0.2609 & 0.2609 & 0.2610 & 0.2611 & 0.2615 & 0.2625
\end{tabular}

